\title{
The Lorentz-violating real scalar field at thermal equilibrium
}

\author{
A. R. Aguirre ${ }^{1, \mathrm{a}}$, G. Flores-Hidalgo ${ }^{1, \mathrm{~b}}$, R. G. Rana ${ }^{1,2, \mathrm{c}}$, E. S. Souza ${ }^{1,3, \mathrm{~d}}$ \\ ${ }^{1}$ Instituto de Física e Química, Universidade Federal de Itajubá, Av. BPS 1303, Itajubá, MG CEP 37500-903, Brazil \\ 2 Centro Brasileiro de Pesquisas Físicas, Rua Dr. Xavier Sigaud 150, Urca, Rio de Janeiro, RJ CEP 22290-180, Brazil \\ ${ }^{3}$ Instituto de Física Gleb Wataghin-UNICAMP, Campinas, SP 13083-859, Brazil
}

Received: 17 March 2021 / Accepted: 15 May 2021 / Published online: 26 May 2021

(C) The Author(s) 2021

\begin{abstract}
In this paper we study Lorentz-violation (LV) effects on the thermodynamics properties of a real scalar field theory due to the presence of a constant background tensor field. In particular, we analyse and compute explicitly the deviations of the internal energy, pressure, and entropy of the system at thermal equilibrium due to the LV contributions. For the free massless scalar field we obtain exact results, whereas for the massive case we perform approximated calculations. Finally, we consider the self interacting $\phi^{4}$ theory, and perform perturbative expansions in the coupling constant for obtaining relevant thermodynamics quantities.
\end{abstract}

\section{Introduction}

Nowadays, it is well-known that symmetries play a fundamental role for describing our modern physical theories. In particular, besides gauge symmetries in the standard model of fundamental particle and interactions, the Lorentz and CPT symmetries are the main building blocks of the theory, and are expected to be respected in any physical situation. However, some years ago, several different theories appeared in the literature, in which apparently such assumption is not longer satisfied [1-6], thereby attracting a great attention in the last few decades both from the theoretical and experimental point of view.

Recently, it has been proposed an interesting generalization of the usual Standard Model that, besides of being consistent with General Relativity and exhibiting all the conventional desirable features of a fundamental physical theory, allows for violations of Lorentz and CPT symmetry $[7,8]$. The main idea of this theory, known as the Standard Model Extension (SME), is that the aforementioned symmetries can

\footnotetext{
a e-mail: alexis.roaaguirre@unifei.edu.br

be-mail: gfloreshidalgo@unifei.edu.br

c e-mail: ranarg@cbpf.br (corresponding author)

de-mail: edsonssouzafis@gmail.com
}

be manifestly broken by incorporating some small constant vector and tensor fields in the theory, generating in this way preferential directions in spacetime. Then, it is expected that such anisotropies in the spacetime should appear as quite small deviations of any physical measurement predicted by a Lorentz-invariant (LI) theory. See Ref. [9] (and references therein) to find updated experimental data that constraining many Lorentz-violating (LV) coefficients in the SME.

The effects of Lorentz violation has been widely investigated in several different scenarios. It is worth mentioning recent studies on the statistical mechanics in LV background [10-14], the Casimir effect [15-20], the Bose-Einstein condensation [21-25], QED sector [26-30], LV theories with boundary conditions [31-34], Chern-Simons-like terms [3539], scattering processes [40-44], geometrical correspondences [45,46], supersymmetric LV models [47-51], as well as many others interesting subjects (see also [52] and references therein for a more exhaustive list of related papers).

On the other hand, finite temperature effects in field theories have been widely studied mainly in the context of cosmological problems [53,54], symmetry restoration in theories with spontaneously broken symmetry [55-57], and more recently in connection with phase transitions in QCD problems related with high energy heavy ion collisions [58-62]. For more articles and textbooks on this subject, see Refs. [63-67].

In the present work, we are interested in investigating the finite temperature effects in a the Lorentz-violating real scalar field theory due to the presence of a constant background tensor contribution. The paper is outlined as follows. In next section, the particle spectrum of the LV model is discussed. In Sect. 3, we discuss the thermal effects in the LV free scalar theory. The interacting case at finite temperature will be analysed in Sect. 4. In Sect. 5, some concluding remarks are presented. Finally, there are some explicit calculations can be found in the appendices. 


\section{Particle spectrum}

In this section, we will discuss the spectrum of a theory with a real scalar field in the presence of a LV background tensor field. Let us consider the following lagrangian density for the real scalar $\phi(x)[7,8]$,

$\mathcal{L}=\frac{1}{2} \partial_{\mu} \phi(x) \partial^{\mu} \phi(x)+\frac{\kappa^{\mu \nu}}{2} \partial_{\mu} \phi(x) \partial_{\nu} \phi(x)-\frac{m^{2}}{2} \phi^{2}$,

where $\kappa_{\mu \nu}$ are dimensionless constant tensor coefficients for the Lorentz-violation that preserves CPT symmetry $[68,69]$. The above model can be identified with the scalar sector of the Standard Model Extension, for which there are phenomenological bounds for the LV parameters in (1), that have been obtained recently from laser interferometry results [70,71]. The space-space components have a superior bound of the order $10^{-18}$ whereas the space-time components have a bound of the order $10^{-12}$ [71]. Although from the phenomenological side the $\mathrm{LV}$ parameters are very small, in this work we will not restrict our calculations to a perturbative treatment on that parameters. Since the model given by (1) is still invariant under space-time translations, the energy and linear momentum will be conserved. In fact, we get respectively,

$$
\begin{aligned}
P^{0}= & H=\frac{1}{2} \int d^{3} \mathbf{x}\left(\Delta^{00} \dot{\phi}^{2}(x)-\Delta^{i j} \partial_{i} \phi(x) \partial_{j} \phi(x)\right. \\
& \left.+m^{2} \phi^{2}(x)\right),
\end{aligned}
$$

and

$\mathbf{P}=-\int d^{3} \mathbf{x} \Delta^{0 \mu} \partial_{\mu} \phi(x) \nabla \phi(x)$,

where $\Delta^{\mu \nu}=\eta^{\mu \nu}+\kappa^{\mu \nu}$ is a modification of the Minkowski metric $\eta^{\mu \nu}$. From Eq. (1) we get the field equation,

$\Delta^{\mu \nu} \partial_{\mu} \partial_{\nu} \phi(x)+m^{2} \phi(x)=0$,

and the canonically conjugate momentum $\pi(x)$, which is given by

$\pi(x)=\Delta^{0 \mu} \partial_{\mu} \phi(x)$.

In order to quantize the theory, we impose the following equal-time commutation relations,

$$
\begin{aligned}
& {[\hat{\phi}(\mathbf{x}, t), \hat{\pi}(\mathbf{y}, t)]=i \delta(\mathbf{x}-\mathbf{y}), \quad[\hat{\phi}(\mathbf{x}, t), \hat{\phi}(\mathbf{y}, t)]=0,} \\
& {[\hat{\pi}(\mathbf{x}, t), \hat{\pi}(\mathbf{y}, t)]=0,}
\end{aligned}
$$

where the field operators satisfy Eqs. (4) and (5). In this way, to solve the field equation for $\hat{\phi}(x)$, we expand in plane waves as follows, $\hat{\phi}(x)=\int \frac{d^{4} p}{(2 \pi)^{4}} \hat{C}(p) e^{-i p x}$.

Substituting in Eq. (4), we get

$\int d^{4} p\left(-\Delta^{\mu v} p_{\mu} p_{v}+m^{2}\right) \hat{C}(p) e^{-i p x}=0$,

from which we find

$\hat{C}(p)=\delta\left(\Delta^{\mu v} p_{\mu} p_{v}-m^{2}\right) \hat{a}(p)$.

Now, by using the above result in Eq. (7), we get,

$\hat{\phi}(x)=\int \widetilde{d p} \frac{1}{\sqrt{\Delta^{00}}}\left(\hat{a}(p) e^{-i p x}+\hat{a}^{\dagger}(p) e^{i p x}\right)$,

where the time-component $p_{0}$ is given by

$$
\begin{aligned}
p_{0} & =\Delta(p)-\frac{\Delta^{0 j} p_{j}}{\Delta^{00}} \\
\Delta(p) & =\sqrt{\left(\frac{\Delta^{0 j} p_{j}}{\Delta^{00}}\right)^{2}+\frac{m^{2}-\Delta^{i j} p_{i} p_{j}}{\Delta^{00}}},
\end{aligned}
$$

and

$\widetilde{d p}=\frac{d^{3} \mathbf{p}}{(2 \pi)^{3} 2 \Delta(p)}$.

Note that we have also redefined $\hat{a}(p)$ in Eq. (10) by a scale factor $\Delta^{00}$, just for convenience. By substituting Eq. (10) in Eq. (5), we get the corresponding expansion for the conjugate momentum,

$\hat{\pi}(x)=-i \int \widetilde{d p} \sqrt{\Delta^{00}} \Delta(p)\left(\hat{a}(p) e^{-i p x}-\hat{a}^{\dagger}(p) e^{i p x}\right)$.

Now, solving for the operators $\hat{a}(p)$ and $\hat{a}^{\dagger}(p)$, we get

$$
\begin{gathered}
\hat{a}(p)=i \int \frac{d^{3} \mathbf{x}}{\sqrt{\Delta^{00}}}\left(\hat{\pi}(x)-i \Delta^{00} \Delta(p) \hat{\phi}(x)\right) e^{i p x}, \\
\hat{a}^{\dagger}(p)=-i \int \frac{d^{3} \mathbf{x}}{\sqrt{\Delta^{00}}}\left(\hat{\pi}(x)+i \Delta^{00} \Delta(p) \hat{\phi}(x)\right) e^{-i p x},
\end{gathered}
$$

which satisfy the following commutation relations,

$$
\begin{aligned}
{\left[\hat{a}(p), \hat{a}^{\dagger}(q)\right] } & =2 \Delta(q)(2 \pi)^{3} \delta(\mathbf{p}-\mathbf{q}), \\
{[\hat{a}(p), \hat{a}(q)] } & =\left[\hat{a}^{\dagger}(p), \hat{a}^{\dagger}(q)\right]=0 .
\end{aligned}
$$

From Eqs. (2), (3), (10) and (13), we obtain the hamiltonian and momentum operators in terms of annihilation and creation operators, namely,

$\hat{H}=\int \widetilde{d p} \frac{p_{0}}{2}\left(\hat{a}^{\dagger}(p) \hat{a}(p)+\hat{a}(p) \hat{a}^{\dagger}(p)\right)$, 
and

$\hat{\mathbf{P}}=\int \widetilde{d p} \frac{\mathbf{p}}{2}\left(\hat{a}^{\dagger}(p) \hat{a}(p)+\hat{a}(p) \hat{a}^{\dagger}(p)\right)$.

Then, we can conclude, from the above expressions and the commutation relations (15), that the spectrum of the theory consists of scalar particles with energy and momentum related by Eq. (11). In fact, we have for the particle energy in terms of LV tensor coefficients $\kappa^{\mu \nu}$, the following expression

$$
p_{0}=\sqrt{\left(\frac{\kappa^{0 j} p_{j}}{1+\kappa^{00}}\right)^{2}+\frac{m^{2}+\mathbf{p}^{2}-\kappa^{i j} p_{i} p_{j}}{1+\kappa^{00}}}-\frac{\kappa^{0 j} p_{j}}{1+\kappa^{00}},
$$

from which it is possible notice that the energy is real, positive and bounded from below whenever the second term within the square root is positive. Otherwise, the particle energy spectrum might exhibit unboundedness or complex values. Considering $\kappa^{00}>-1$, these issues can be ruled out if the eigenvalues of the matrix $\kappa^{i j}$ are less than the unity, i.e. $\left|\kappa^{i j}\right|<<1$, which is naturally expected for a realistic theory. For other values of $\kappa^{i j}$ it is possible to fix some suitable limits in order to have a well defined particle spectrum. For instance, by considering the case in which all diagonal terms are equal to $\kappa^{11}$, and respectively all offdiagonal terms are $\kappa^{12}$, we find that the suitable conditions are $\kappa^{11}-\kappa^{12}<1$, and $\kappa^{11}+2 \kappa^{12}<1$. On the other hand, by considering arbitrary diagonal terms, $\kappa^{11}, \kappa^{22}, \kappa^{33}$, and all the off-diagonal terms vanishing except for $\kappa^{12}=\kappa^{21}$, we find a well behaved particle spectrum if $\kappa^{33}<1, \kappa^{11}+\kappa^{22}<1$ and $\kappa^{11}+\kappa^{22}+\left(\kappa^{12}\right)^{2}-\kappa^{11} \kappa^{22}<1$. Also, tachyonic modes must appear whenever the argument in square root of (18) is negative. Therefore to avoid that modes it is sufficient that the eigenvalues of the matrix quadratic form in $p_{i} p_{j}$ be non negative. If the Lorentz violating parameter are not so large this will be always the case.

Thus, in order to avoid those sort of undesirable behaviours for particle spectrum, from now on we will assume that the magnitude of the constant tensor components $\kappa^{\mu \nu}$ are not so large, and proper conditions guaranteeing a well-defined particle spectrum are satisfied.

\section{Thermal behaviour for the free scalar field}

In this section we analyze the effects and contributions of the LV terms on the thermodynamics of the free real scalar field. Let us start from the partition function,

$$
Z=\int \mathcal{D} \phi \exp \left(-\int_{0}^{\beta} d \tau \int d^{3} \mathbf{x} \mathcal{L}_{E}\right)
$$

where $\beta=T^{-1}$ is the inverse of the temperature, ${ }^{1}$ and $\mathcal{L}_{E}$ is the Euclidean density lagrangian of the theory with $\mathrm{LV}$ terms, obtained from the prescription $t \rightarrow i \tau$, and given by

$\mathcal{L}_{E}=\frac{1}{2} \bar{\Delta}_{\mu \nu} \partial_{\mu} \phi(x) \partial_{\nu} \phi(x)+U(\phi)$,

with $U(\phi)$ contains the mass and possible interaction terms, and

$\bar{\Delta}_{00}=1+\kappa^{00}, \quad \bar{\Delta}_{0 j}=-i \kappa^{0 j}, \quad \bar{\Delta}_{i j}=\delta^{i j}-\kappa^{i j}$.

It is worth pointing out that the integration in Eq. (19) must be computed over all periodic field configurations, i.e. $\phi(\mathbf{x}, \tau+$ $\beta)=\phi(\mathbf{x}, \beta)$. Now, the thermal Green functions, defined as the thermal expectation values of imaginary time-ordered products of field operators, are given by

$$
\begin{aligned}
\left\langle\phi\left(x_{1}\right) \ldots \phi\left(x_{n}\right)\right\rangle= & \frac{1}{Z} \int \mathcal{D} \phi \phi\left(x_{1}\right) \ldots \phi\left(x_{n}\right) \\
& \times \exp \left(-\int_{0}^{\beta} d \tau \int d^{3} \mathbf{x} \mathcal{L}_{E}\right), \\
= & \left.\frac{1}{Z(j)} \frac{\delta^{n} Z(j)}{\delta j\left(x_{1}\right) \ldots \delta j\left(x_{n}\right)}\right|_{j=0},
\end{aligned}
$$

where $Z(j)$ denotes the thermal generating functional,

$Z(j)=\int \mathcal{D} \phi \exp \left(-\int_{0}^{\beta} d \tau \int d^{3} \mathbf{x}\left(\mathcal{L}_{E}-j \phi\right)\right)$

Firstly, we will focus on the free case, $U(\phi)=m^{2} \phi^{2} / 2$. In that case the associated thermal propagator $D(x, y)$ for the free real scalar field, which corresponds to the two-point Green function (22), follows the equation,

$\bar{\square}(\mathbf{x}, \tau)=\delta(\mathbf{x}) \delta(\tau)$,

where $\bar{\square}=\left(-\bar{\Delta}_{\mu \nu} \partial_{\mu} \partial_{\nu}+m^{2}\right)$, and we have taken $y=0$ without loss of generality. Now, considering periodic boundary conditions in the imaginary time, we can write $D(\mathbf{x}, \tau)$, as follows

$D(\mathbf{x}, \tau)=\frac{1}{\beta} \sum_{n=-\infty}^{\infty} \int \frac{d^{3} \mathbf{p}}{(2 \pi)^{3}} e^{-i\left(\omega_{n} \tau-\mathbf{p . x}\right)} \widetilde{D}\left(\mathbf{p}, \omega_{n}\right)$,

where $\omega_{n}=2 \pi n / \beta, n=0, \pm 1, \pm 2, \ldots$ are the Matsubara frequencies. Substituting Eq. (25) in Eq. (24), we get the thermal propagator in the Fourier space,

$\widetilde{D}\left(\mathbf{p}, \omega_{n}\right)=\frac{1}{\bar{\Delta}_{00} \omega_{n}^{2}-2 \omega_{n} \bar{\Delta}_{0 j} p_{j}+\bar{\Delta}_{i j} p_{i} p_{j}+m^{2}}$.

\footnotetext{
${ }^{1}$ Here we are using the usual convention in which the Boltzmann constant is $k_{B}=1$, as well as $c=\hbar=1$.
} 
Notice that this representation of the thermal propagator is complex-valued since $\bar{\Delta}_{0 j}$ is imaginary. However, this is not a problem since its representation in the configuration space (25) is real, as it is expected for a real field.

Now, we will compute the partition function (19), from which we will obtain thermodynamic quantities as the internal energy, pressure, and entropy of the system. From Eq. (19) we find

$$
Z_{0}=\int \mathcal{D} \phi \exp \left(-\frac{1}{2} \int_{0}^{\beta} d \tau \int d^{3} \mathbf{x} \phi(x) \bar{\square} \phi(x)\right),
$$

where the zero lower label stands for the free case. As usual, taking the system inside a box of volume $V$, and considering periodic boundary conditions, we can expand the field as follows

$\phi(\tau, \mathbf{x})=\sqrt{\frac{\beta}{V}} \sum_{n=-\infty}^{\infty} \sum_{\mathbf{p}} \tilde{\phi}_{n}(\mathbf{p}) e^{-i\left(\omega_{n} \tau-\mathbf{p} \cdot \mathbf{x}\right)}$.

Now, by using the orthogonality relations

$\int_{0}^{\beta} d \tau \int d^{3} \mathbf{x} e^{i\left(\omega_{n}-\omega_{l}\right) \tau-i(\mathbf{p}-\mathbf{q}) \cdot \mathbf{x}}=\beta V \delta_{n l} \delta_{\mathbf{p q}}$,

and $\tilde{\phi}_{-n}(-\mathbf{p})=\tilde{\phi}_{n}^{*}(\mathbf{p})$, we can write

$$
\begin{aligned}
Z_{0}= & N \int \prod_{n} \prod_{\mathbf{p}} d \tilde{\phi}_{n}(\mathbf{p}) \\
& \times \exp \left[-\frac{\beta^{2}}{2} \sum_{n=-\infty}^{\infty} \sum_{\mathbf{p}} \widetilde{D}^{-1}\left(\mathbf{p}, \omega_{n}\right)\left|\tilde{\phi}_{n}(\mathbf{p})\right|^{2}\right] .
\end{aligned}
$$

The integrals above can be computed by writing $\tilde{\phi}_{n}(\mathbf{p})$ in polar form, and integrating in the corresponding modulus. Doing that, we obtain

$$
\begin{aligned}
Z_{0}= & N^{\prime} \prod_{n \mathbf{p}}\left[\beta ^ { 2 } \left(\bar{\Delta}_{00} \omega_{n}^{2}-2 \bar{\Delta}_{0 j} \omega_{n} p_{j}\right.\right. \\
& \left.\left.+\bar{\Delta}_{i j} p_{i} p_{j}+m^{2}\right)\right]^{-1 / 2},
\end{aligned}
$$

where contributions from the phase integrals have been incorporated in $N^{\prime}$, and therefore we find

$$
\begin{aligned}
\ln Z_{0}= & \ln N^{\prime}-\frac{1}{2} \sum_{n=-\infty}^{\infty} \sum_{\mathbf{p}} \ln \left(4 \pi^{2} n^{2}-4 \pi \beta \frac{\bar{\Delta}_{0 j}}{\bar{\Delta}_{00}} n p_{j}\right. \\
& \left.+\beta^{2} \frac{\bar{\Delta}_{i j}}{\bar{\Delta}_{00}} p_{i} p_{j}+\beta^{2} \frac{m^{2}}{\bar{\Delta}_{00}}\right) .
\end{aligned}
$$

Now, by summing over $n$, and noting that $N^{\prime}$ is independent from the LV coefficients, we can proceed by using a similar prescription as in the Lorentz invariant case (see Appendix A), and then we have $\ln Z_{0}=-V \int \frac{d^{3} \mathbf{p}}{(2 \pi)^{3}}\left[\frac{1}{2} \beta p_{0}+\ln \left(1-e^{-\beta p_{0}}\right)\right]$,

where $p_{0}$ given in Eq. (18) is the particle energy spectrum. The first term in the above expression is divergent and represent the vacuum energy, which has been analyzed recently in connection with the Casimir energy in Refs. [15-18]. Since we are interested only in studying thermal properties, we will disregard that term from now on. We notice also that as in the LI case, the expression (33) scales with the volume $V$, which is a consequence of translational invariance of the system. To compute explicitly the expression (33), we will consider separately the massless and the massive case.

\subsection{The free massless case}

The integral (33) can be computed exactly for the massless case. In this case the particle energy, which we will denote as $\tilde{p}_{0}$, takes the following form,

$$
\begin{aligned}
\tilde{p}_{0} & =\sqrt{p_{i} A_{i j} p_{j}}-\frac{\kappa^{0 j} p_{j}}{1+\kappa^{00}}, \\
A_{i j} & =\frac{\kappa^{0 i} \kappa^{0 j}}{\left(1+\kappa^{00}\right)^{2}}+\frac{\delta_{i j}-\kappa^{i j}}{1+\kappa^{00}} .
\end{aligned}
$$

Now, we introduce a matrix $M_{i j}$ that diagonalizes $A_{i j}$, namely $M^{-1} A M=\operatorname{diag}\left(a_{1}, a_{2}, a_{3}\right)$, where $a_{1}, a_{2}$ and $a_{3}$, are the eigenvalues of matrix $A_{i j}$. By defining new variables

$p_{i}=\frac{1}{\beta \sqrt{a_{j}}} M_{i j} \bar{p}_{j}$,

and disregarding the vacuum energy contribution, we get for (33),

$\left.\ln Z_{0}\right|_{m=0}=-\frac{V}{\beta^{3}} \frac{|\operatorname{det} M|}{|\operatorname{det} A|^{1 / 2}} \int \frac{d^{3} \overline{\mathbf{p}}}{(2 \pi)^{3}} \ln \left[1-e^{-\bar{p}+\mathbf{b} \cdot \overline{\mathbf{p}}}\right]$,

where $\bar{p}=|\overline{\mathbf{p}}|$, and the components of $\mathbf{b}$ are

$b^{j}=\frac{\kappa^{0 i} M_{i j}}{\sqrt{a_{j}}\left(1+\kappa^{00}\right)}$.

The integral (36) can be computed straightforwardly in spherical coordinates, obtaining the following final result

$\left.\ln Z_{0}\right|_{m=0}=V \frac{\pi^{2}}{90\left(1-b^{2}\right)^{2}} \frac{|\operatorname{det} M|}{|\operatorname{det} A|^{1 / 2}} \beta^{-3}$,

where $b=|\mathbf{b}|$. From this result we can determine the internal energy,

$U=V \frac{\pi^{2}}{30\left(1-b^{2}\right)^{2}} \frac{|\operatorname{det} M|}{|\operatorname{det} A|^{1 / 2}} T^{4}$, 
and the pressure,

$P=\frac{\pi^{2}}{90\left(1-b^{2}\right)^{2}} \frac{|\operatorname{det} M|}{|\operatorname{det} A|^{1 / 2}} T^{4}$,

from which we obtain the relation,

$P V=\left.T \ln Z_{0}\right|_{m=0}=\frac{U}{3}$,

where the dependence on the LV parameters is hidden. This happens because in the masless case, the LV model posseses a particle energy spectrum (34) which is a homogeneous function of degree one in the momentum variable, as in the LI case.

However, as we will see in the next subsection, that behavior is a peculiarity of the massless case. Now, considering the mean number of particles

$N=V \int \frac{d^{3} \mathbf{p}}{(2 \pi)^{3}} \frac{1}{e^{\tilde{p}_{0} \beta}-1}$,

and using (34), we obtain

$N=V \frac{1}{\pi^{2}\left(1-b^{2}\right)^{2}} \frac{|\operatorname{det} M|}{|\operatorname{det} A|^{1 / 2}} \zeta(3) T^{3}$.

And finally for the entropy, we get

$S=V \frac{2 \pi^{2}}{45\left(1-b^{2}\right)^{2}} \frac{|\operatorname{det} M|}{|\operatorname{det} A|^{1 / 2}} T^{3}$.

Since all of the above thermodynamics quantities are derived from Eq. (38), they are all modified in relation to the Lorentzinvariant case by a global multiplicative factor. Also, from Eq. (43) we see that the mean number of particles is modified by the same factor. From this behaviour, we can conclude in general that for small violating parameters $\kappa$, the thermodynamics quantities increase or decrease according to whether these parameters are positive or negative. To illustrate that, we can evaluate explicitly Eq. (38) for some specific situations consistent with the conditions discussed at the end of Sect. 2. In the first scenario, let us consider all the $\kappa$ components as null except for $\kappa^{00}$ and $\kappa^{11}$. Then we obtain

$\left.\ln Z_{0}\right|_{m=0}=V \frac{\pi^{2}}{90} \frac{\left(1+\kappa^{00}\right)^{3 / 2}}{\left(1-\kappa^{11}\right)^{1 / 2}} \beta^{-3}$.

In the second scenario, we consider $\kappa^{02}$ as the only non-null component. So, we get,

$\left.\ln Z_{0}\right|_{m=0}=V \frac{\pi^{2}}{90}\left(1+\left(\kappa^{02}\right)^{2}\right)^{3 / 2} \beta^{-3}$.
For the last scenario, we choose $\kappa^{12}$ as the only non-null component. Hence,

$\left.\ln Z_{0}\right|_{m=0}=V \frac{\pi^{2}}{45} \frac{1}{\left(1-3\left(\kappa^{12}\right)^{2}\right)^{1 / 2}} \beta^{-3}$.

In general, we notice that the global multiplicative factor, which contain all the corrections, can in principle increase or decrease the standard result, i.e. the one obtained in the Lorentz-invariant case, by a linear or quadratic contribution of the $\kappa$ components, depending on whether they are diagonal or off-diagonal terms. Of course, by considering that all the $\kappa$ components are non-null, we will obtain a more complicated form which will contain higher powers of them. However, those additional terms will not represent any significant contributions. In fact, if we expand the multiplicative factor in powers of these components, it will be enough to keep up to linear order for practical purposes.

As is well known, at linear order in the LV parameters, the coordinate transformation $x^{\prime \mu}=\left(\delta_{v}^{\mu}-\frac{1}{2} \kappa_{v}^{\mu}\right) x^{\nu}$ reduces the LV density Lagrangian (1) to the usual one up to a Jacobian factor [72]. In this way, all the results in the LV case can be obtained from the LI case, taking the space-time volume multiplied by the corresponding Jacobian. At finite temperature, the Euclidean space-time volume is given by $\beta V$, and is this factor that must be multiplied by the aforementioned Jacobian. In the case in which $\kappa^{0 j}=0$, the coordinate transformation above does no mix space and time coordinates and in this case the inverse temperature $\beta$ and the spatial volume $V$ transforms independently, as

$\beta \rightarrow\left(1-\frac{1}{2} \kappa_{0}^{0}\right) \beta, \quad V \rightarrow\left(1+\frac{1}{2}\left(\kappa^{11}+\kappa^{22}+\kappa^{33}\right)\right) V$

In this way, starting from the known result for the partition function in the massless scalar LI case, namely

$\left.\ln Z_{0}\right|_{m=0}=\frac{\pi^{2}}{90} V \beta^{-3}$

and using correspondences in Eq. (48), we get

$\left.\ln Z_{0}\right|_{m=0}=\frac{\pi^{2}}{90}\left[1+\frac{3}{2} k^{00}+\frac{1}{2}\left(\kappa^{11}+\kappa^{22}+\kappa^{33}\right)\right] V \beta^{-3}$,

which coincides, up to linear order in the LV parameters $\kappa^{\mu \nu}$, with the corresponding expressions given by Eqs. (45)-(47). The above check gives us confidence about the consistency of our results.

\subsection{The free massive case}

Now, we will evaluate the partition function for the free massive case. Since we are not able to perform exactly the integral 
(33), we are going to consider the high temperature regime instead, i.e. $m / T \ll 1(m \beta \ll 1)$, and then we will expand the thermodynamics quantities in terms of this parameter. First of all, we expand $p_{0}(18)$ as follows

$p_{0} \simeq \tilde{p}_{0}+\delta p_{0}$,

with

$\delta p_{0}=\frac{m^{2}}{2\left(1+\kappa^{00}\right) \sqrt{p_{i} A_{i j} p_{j}}}$,

where $\tilde{p}_{0}$ and $A_{i j}$ are given in Eq. (34). Substituting the above approximation in the integral (33), and expanding up to first order in $\delta p_{0}$, we have

$\ln Z_{0}=\left.\ln Z_{0}\right|_{m=0}-V \beta \int \frac{d^{3} \mathbf{p}}{(2 \pi)^{3}} \frac{\delta p_{0}}{e^{\beta \tilde{p}_{0}}-1}+\cdots$.

Now, by using the redefinition (35), we obtain

$$
\begin{aligned}
\ln Z_{0}= & \left.\ln Z_{0}\right|_{m=0}-V \beta^{-1} m^{2} \frac{|\operatorname{det} M|}{2|\operatorname{det} A|^{1 / 2}\left(1+\kappa^{00}\right)} \\
& \times \int \frac{d^{3} \overline{\mathbf{p}}}{(2 \pi)^{3}} \frac{1}{\bar{p}\left(e^{\bar{p}-\mathbf{b} \cdot \overline{\mathbf{p}}}-1\right)}+\cdots .
\end{aligned}
$$

Now, performing the final integration, we finally get,

$$
\begin{aligned}
\ln Z_{0}= & V \frac{\pi^{2}}{90\left(1-b^{2}\right)^{2}} \frac{|\operatorname{det} M|}{|\operatorname{det} A|^{1 / 2}} \beta^{-3} \\
& \times\left(1-\frac{15\left(1-b^{2}\right)}{4 \pi^{2}\left(1+\kappa^{00}\right)} \beta^{2} m^{2}+\mathcal{O}\left((\beta m)^{4}\right)\right) .
\end{aligned}
$$

As it was done for the massless case, from the above result we can determine the internal energy and pressure, namely

$$
\begin{aligned}
U= & V \frac{\pi^{2}}{30\left(1-b^{2}\right)^{2}} \frac{|\operatorname{det} M|}{|\operatorname{det} A|^{1 / 2}} T^{4} \\
& \times\left(1-\frac{5\left(1-b^{2}\right)}{4 \pi^{2}\left(1+\kappa^{00}\right)} m^{2} T^{-2}+\mathcal{O}\left((m / T)^{4}\right)\right), \\
P= & \frac{\pi^{2}}{90\left(1-b^{2}\right)^{2}} \frac{|\operatorname{det} M|}{|\operatorname{det} A|^{1 / 2}} T^{4} \\
& \times\left(1-\frac{15\left(1-b^{2}\right)}{4 \pi^{2}\left(1+\kappa^{00}\right)} m^{2} T^{-2}+\mathcal{O}\left((m / T)^{4}\right)\right),
\end{aligned}
$$

which satisfy the relation

$$
\begin{aligned}
P V & =T \ln Z_{0} \\
& =\frac{U}{3}\left(1-\frac{10\left(1-b^{2}\right)}{4 \pi^{2}\left(1+\kappa^{00}\right)} m^{2} T^{-2}+\mathcal{O}\left((m / T)^{4}\right)\right) .
\end{aligned}
$$

Also, we find that the entropy is given by

$$
\begin{aligned}
S= & V \frac{\pi^{2}}{90\left(1-b^{2}\right)^{2}} \frac{|\operatorname{det} M|}{|\operatorname{det} A|^{1 / 2}} T^{3} \\
& \times\left(4-\frac{15\left(1-b^{2}\right)}{2 \pi^{2}\left(1+\kappa^{00}\right)} m^{2} T^{-2}+\mathcal{O}\left((m / T)^{4}\right)\right),
\end{aligned}
$$

and the mean number of particles reads

$$
\begin{aligned}
N= & \frac{V|\operatorname{det} M| T^{3}}{\pi^{2}|\operatorname{det} A|^{1 / 2}}\left[\zeta(3)+\frac{m^{2} T^{-2}}{4\left(1+\kappa^{00}\right)}\right. \\
& \left.+\ln \sinh \left(\frac{m}{2 \sqrt{\left(1+\kappa^{00}\right)} T}\right)+\mathcal{O}\left((m / T)^{4}\right)\right],
\end{aligned}
$$

where it has been considered only the case $b=0$, for simplicity (see Appendix B for more details).

Now, as it was done in the massless case, we will analyze the effect of LV terms on the expression (54). Let us first consider that only $\kappa^{00} \neq 0$, and the rest of the components are null. Up to second order in $\beta m$, we find,

$\ln Z_{0}=\frac{\pi^{2}}{90} \frac{V}{\beta^{3}}\left(1+\kappa^{00}\right)^{3 / 2}\left(1-\frac{15}{4 \pi^{2}\left(1+\kappa^{00}\right)} \beta^{2} m^{2}\right)$.

If we consider that only the $\kappa^{11}$ component is different from zero, we obtain

$\ln Z_{0}=\frac{\pi^{2}}{90} \frac{V}{\beta^{3}}\left(1+\kappa^{11}\right)^{-1 / 2}\left(1-\frac{15}{4 \pi^{2}} \beta^{2} m^{2}\right)$.

We note that both results suffer an explicit global effect from the LV parameters, but only the term $\kappa^{00}$ appears explicitly in the term proportional to mass. For $0<\kappa^{00}<1$, the global term of pressure and energy increase, but the term proportional to the mass decreases these quantities. For $-1<\kappa^{00}<0$, the global and the mass terms decrease the pressure and energy. Comparing these two cases, we can see that for the positive $\kappa^{00}$, the mass term decrease these thermodynamics quantities more than the negative $\kappa^{00}$ case. In the case of $\kappa^{11} \neq 0$, the Lorentz-violation term appears only as a global term. For negative $\kappa^{11}$, the pressure and energy decreases more than the positive $\kappa^{11}$ case. On the other hand, when considering the cross-terms $\kappa^{0 i}$ and $\kappa^{i j}$, we conclude that they appear, at least, in quadratic order in the Eq. (54). Regarding the state equations, we see that, unlike the massless case, there is an explicit effect of the LV terms, even when written in terms of $N$. 


\section{The thermal behaviour for the interacting scalar field}

In this section we consider the study of the thermal properties of the real scalar interacting field, with Euclidean lagrangian given by (20), where

$U(\phi)=\frac{m^{2}}{2} \phi^{2}+\frac{\lambda}{4 !} \phi^{4}$.

To compute the partition function perturbatively, we decompose the Euclidean action in the free and interacting part,

$S_{E}=S_{0}+S_{I}$,

where $S_{0}$ is the free Euclidean action and $S_{I}$ is given by the integral of the last term in (62). In this way, expanding the interacting part we have from (19),

$\ln Z=\ln Z_{0}+\ln Z_{I}$,

where $Z_{0}$ is the free partition function considered and evaluated in the last section, and $\ln Z_{I}$ is given by,

$$
\begin{aligned}
\ln Z_{I}= & \ln \left(1+\frac{1}{Z_{0}} \sum_{n=1}^{\infty} \frac{(-1)^{n}}{n !} \int \mathcal{D} \phi e^{-S_{0}}\left(S_{I}\right)^{n}\right) \\
= & \ln \left(1+\sum_{n=1}^{\infty} \frac{(-\lambda)^{n}}{4^{n} n !} \int d x_{1} \ldots \int d x_{n}\right. \\
& \left.\times\left\langle\phi^{4}\left(\tau_{1}, \mathbf{x}_{1}\right) \ldots \phi^{4}\left(\tau_{n}, \mathbf{x}_{n}\right)\right\rangle\right) .
\end{aligned}
$$

In above expression, we have used the shorthand notation $d x$ to denote $d \tau d^{3} \mathbf{x}$ and

$\langle\ldots\rangle=\frac{1}{Z_{0}} \int \mathcal{D} \phi \ldots e^{-S_{0}}$,

that we can compute using the Wick theorem with the help of the free propagator (25) and (26). In this way it is possible to show that (65) is given schematically by the connected vacuum graphs, but replacing the zero temperature propagator by the thermal propagator, and also the vertex $-i \lambda$ by $-\lambda$. So, at first order in the coupling constant, Eq. (65) reads

$$
\begin{aligned}
\ln Z_{I} & =-\frac{\lambda}{4 !} \int_{0}^{\beta} d \tau \int d^{3} \mathbf{x}\left\langle\phi^{4}(x)\right\rangle \\
& =-\frac{\lambda}{8} \int_{0}^{\beta} d \tau \int d^{3} \mathbf{x}[D(\mathbf{0}, 0)]^{2} .
\end{aligned}
$$

Using (25) and (26), the above expression can be written as

$\ln Z_{I}=-V \frac{\lambda}{8 \beta}\left(\sum_{n=-\infty}^{\infty} \int \frac{d^{3} \mathbf{p}}{(2 \pi)^{3}} \widetilde{D}\left(\mathbf{p}, \omega_{n}\right)\right)^{2}$.
Now, by replacing the Matsubara frequencies in (68), and summing in $n$, we get

$\ln Z_{I}=-V \beta \frac{\lambda}{8}\left[\int \frac{d^{3} \mathbf{p}}{(2 \pi)^{3}}\left(\mathcal{F}_{+}(p)+\mathcal{F}_{-}(p)\right)\right]^{2}$,

where

$\mathcal{F}_{ \pm}(p)=\frac{1}{4 \mathcal{R}} \operatorname{coth}\left(\frac{\beta}{2 \bar{\Delta}_{00}}\left(\mathcal{R} \pm i \bar{\Delta}_{0 j} p_{j}\right)\right)$,

with

$\mathcal{R}=\sqrt{\left(i \bar{\Delta}_{0 j} p_{j}\right)^{2}+\bar{\Delta}_{00}\left(\bar{\Delta}_{i j} p_{i} p_{j}+m^{2}\right)}$.

By performing the transformation $\mathbf{p} \rightarrow-\mathbf{p}$, we can show that $\mathcal{F}_{+}$and $\mathcal{F}_{-}$within the integral (69) are equal, and then using (21) we get

$$
\begin{aligned}
\ln Z_{I}= & -V \beta \frac{\lambda}{32}\left[\int \frac{d^{3} \mathbf{p}}{(2 \pi)^{3}} \frac{\operatorname{coth}\left(\beta p_{0} / 2\right)}{\left(1+\kappa^{00}\right) p_{0}+\kappa^{0 j} p_{j}}\right]^{2} \\
= & -V \beta \frac{\lambda}{32}\left[\int \frac{d^{3} \mathbf{p}}{(2 \pi)^{3}} \frac{1}{\left(1+\kappa^{00}\right) p_{0}+\kappa^{0 j} p_{j}}\right. \\
& \left.+\frac{2}{\left[\left(1+\kappa^{00}\right) p_{0}+\kappa^{0 j} p_{j}\right]\left(e^{\beta p_{0}}-1\right)}\right]^{2},
\end{aligned}
$$

where $p_{0}$ is the free particle energy, given by (18). The first term in (72), is ultraviolet divergent but linear in the inverse temperature $\beta$. Such term gives the first-order vacuum energy correction and has been treated recently in connection to the radiative corrections to the Casimir energy [19]. Also, since we are interested only in studying the thermal properties of the system, from now on we disregard such contribution as we did in the free case. In this way, we get for the finite temperature dependent part,

$$
\ln Z_{I}=-\frac{\lambda \beta V}{8}\left[\int \frac{d^{3} \mathbf{p}}{(2 \pi)^{3}} \frac{\left(e^{\beta p_{0}}-1\right)^{-1}}{\left[\left(1+\kappa^{00}\right) p_{0}+\kappa^{0 j} p_{j}\right]}\right]^{2} .
$$

\subsection{The massless case}

For the massless case, the above integral can be done exactly. In this case, by using (34) in (73), and performing the change of variable (35), we have

$$
\begin{aligned}
\left.\ln Z_{I}\right|_{m=0}= & -V \beta \frac{\lambda}{8}\left[\frac{\beta^{-2}|\operatorname{det} M|}{|\operatorname{det} A|^{1 / 2}\left(1+\kappa^{00}\right)}\right]^{2} \\
& \times\left[\int \frac{d^{3} \overline{\mathbf{p}}}{(2 \pi)^{3}} \frac{1}{\bar{p}\left(e^{\bar{p}-\mathbf{b} \cdot \overline{\mathbf{p}}}-1\right)}\right]^{2} .
\end{aligned}
$$

Now, using polar coordinates, we obtain

$$
\left.\ln Z_{I}\right|_{m=0}=-V \beta^{-3} \frac{\lambda}{2^{7} \cdot 9} \frac{|\operatorname{det} M|^{2}}{|\operatorname{det} A|\left(1+\kappa^{00}\right)^{2}\left(1-b^{2}\right)^{2}} .
$$


Replacing Eqs. (38) and (75) in Eq. (64), we have for the massless interacting partition function

$$
\begin{aligned}
\left.\ln Z\right|_{m=\sigma}= & V \frac{|\operatorname{det} M|}{\left(1-b^{2}\right)^{2}|\operatorname{det} A|^{1 / 2}} \beta^{-3} \\
& \times\left(\frac{\pi^{2}}{90}-\frac{\lambda}{2^{7} \cdot 9} \frac{|\operatorname{det} M|}{|\operatorname{det} A|^{1 / 2}\left(1+\kappa^{00}\right)^{2}}+\cdots\right),
\end{aligned}
$$

from which the internal energy and pressure can be obtained,

$$
\begin{aligned}
U= & V \frac{|\operatorname{det} M|}{\left(1-b^{2}\right)^{2}|\operatorname{det} A|^{1 / 2}} T^{4} \\
& \times\left(\frac{\pi^{2}}{30}-\frac{\lambda}{2^{7} \cdot 3} \frac{|\operatorname{det} M|}{|\operatorname{det} A|^{1 / 2}\left(1+\kappa^{00}\right)^{2}}+\cdots\right),
\end{aligned}
$$

and

$$
\begin{aligned}
P= & \frac{|\operatorname{det} M|}{\left(1-b^{2}\right)^{2}|\operatorname{det} A|^{1 / 2}} T^{4} \\
& \times\left(\frac{\pi^{2}}{90}-\frac{\lambda}{2^{7} \cdot 9} \frac{|\operatorname{det} M|}{|\operatorname{det} A|^{1 / 2}\left(1+\kappa^{00}\right)^{2}}+\cdots\right) .
\end{aligned}
$$

Also, for the entropy, we get

$$
\begin{aligned}
S= & V \frac{|\operatorname{det} M|}{\left(1-b^{2}\right)^{2}|\operatorname{det} A|^{1 / 2}} T^{3} \\
& \times\left(\frac{2 \pi^{2}}{45}-\frac{\lambda}{2^{5} \cdot 9} \frac{|\operatorname{det} M|}{|\operatorname{det} A|^{1 / 2}\left(1+\kappa^{00}\right)^{2}}+\cdots\right) .
\end{aligned}
$$

From (77) and (78) we see that at first order in the coupling constant $P V=U / 3$, as in the free massless case.

We will now consider some specific cases. When only $\kappa^{00} \neq 0$, it can be noticed that the behaviour of the above thermodynamics quantities is similar to the massive case without interaction, where $\lambda$ plays the role of the mass. However, each one of these effects has different magnitude. On the other hand, by considering only $\kappa^{11} \neq 0$, unlike the massive case without interaction, the thermodynamics quantities decrease by the interaction term $\lambda$. However, the similarity with the massive case without interaction comes from the global term that always decreases the pressure and the energy.

\subsection{The massive case}

In this situation the integrand in (73) can not be performed exactly. As in the free massive case we expand in the parameter $\beta m$, and then our result will be valid for small values of $\beta m$ (see the full details of the calculation in Appendix C). According to the free case, we only consider the first-order correction, and then we have for the interacting massive partition function,

$$
\begin{aligned}
\ln Z= & V \frac{\pi^{2}}{90\left(1-b^{2}\right)^{2}} \frac{|\operatorname{det} M|}{|\operatorname{det} A|^{1 / 2}} \beta^{-3} \\
& \times\left(1-\frac{15\left(1-b^{2}\right)}{4 \pi^{2}\left(1+\kappa^{00}\right)} \beta^{2} m^{2}+\mathcal{O}\left((\beta m)^{4}\right)\right) \\
& -V \frac{\lambda}{2^{7} \cdot 9} \frac{|\operatorname{det} M|^{2}}{\left(1-b^{2}\right)^{2}|\operatorname{det} A|\left(1+\kappa^{00}\right)^{2}} \beta^{-3} \\
& \times\left(1-3 \frac{\left(1-b^{2}\right) \tilde{b}}{\pi\left(1+\kappa^{00}\right)^{1 / 2}} \beta m+\cdots\right),
\end{aligned}
$$

where $\tilde{b}$ is given by

$\tilde{b}=(1+b)^{-1 / 2}+(1-b)^{-1 / 2}$.

In the second line in (80) we have disregarded quadratic terms in $\beta m$, since being the constant coupling $\lambda$ sufficiently small, we expect that crossing terms of the type $\lambda(\beta m)^{2}$ are also very small compared with the free contribution. Now, from (80) we get for the internal energy, the pressure and entropy, the following expressions

$$
\begin{aligned}
U= & V \frac{\pi^{2}}{30\left(1-b^{2}\right)^{2}} \frac{|\operatorname{det} M|}{|\operatorname{det} A|^{1 / 2}} T^{4} \\
& \times\left(1-\frac{5\left(1-b^{2}\right)}{4 \pi^{2}\left(1+\kappa^{00}\right)} m^{2} T^{-2}+\cdots\right) \\
& -V \frac{\lambda}{2^{7} \cdot 3} \frac{|\operatorname{det} M|^{2}}{\left(1-b^{2}\right)^{2}|\operatorname{det} A|\left(1+\kappa^{00}\right)^{2}} T^{4} \\
& \times\left(1-2 \frac{\left(1-b^{2}\right) \tilde{b}}{\pi\left(1+\kappa^{00}\right)^{1 / 2}} m T^{-1}+\cdots\right), \\
P= & \frac{\pi^{2}}{90\left(1-b^{2}\right)^{2}} \frac{|\operatorname{det} M|}{|\operatorname{det} A|^{1 / 2}} T^{4} \\
& \times\left(1-\frac{15\left(1-b^{2}\right)}{4 \pi^{2}\left(1+\kappa^{00}\right)} m^{2} T^{-2}+\cdots\right) \\
& -\frac{\lambda}{2^{7} \cdot 9} \frac{|\operatorname{det} M|^{2}}{\left(1-b^{2}\right)^{2}|\operatorname{det} A|\left(1+\kappa^{00}\right)^{2}} T^{4} \\
& \times\left(1-3 \frac{\left(1-b^{2}\right) \tilde{b}}{\pi\left(1+\kappa^{00}\right)^{1 / 2}} m T^{-1}+\cdots\right),
\end{aligned}
$$

and

$$
\begin{aligned}
S= & V \frac{2 \pi^{2}}{45\left(1-b^{2}\right)^{2}} \frac{|\operatorname{det} M|}{|\operatorname{det} A|^{1 / 2}} T^{3} \\
& \times\left(1-\frac{15\left(1-b^{2}\right)}{8 \pi^{2}\left(1+\kappa^{00}\right)} m^{2} T^{-2}+\cdots\right) \\
& -V \frac{\lambda}{2^{5} \cdot 9} \frac{|\operatorname{det} M|^{2}}{\left(1-b^{2}\right)^{2}|\operatorname{det} A|\left(1+\kappa^{00}\right)^{2}} T^{3} \\
& \times\left(1-\frac{9}{4} \frac{\left(1-b^{2}\right) \tilde{b}}{\pi\left(1+\kappa^{00}\right)^{1 / 2}} m T^{-1}+\cdots\right) .
\end{aligned}
$$

We notice from these results that when $\kappa^{00}$ is the only non-null coefficient, and $-1<\kappa^{00}<0$, the global factor 
decrease the thermodynamics quantities. On the contrary, when $0<\kappa^{00}<1$, the global factor will increases these quantities. In its turn, the coefficient $\beta^{2} m^{2}$ will gives also a decreasing contribution, whereas the term proportional to $\lambda \beta m$ will give an increasing contribution. However, the magnitude of such contributions will depend on the sign of $\kappa^{00}$.

On the other hand, by considering that $\kappa^{11}$ is the only non-vanishing LV coefficient, we see that in the regime $-1<\kappa^{11}<0$ the global factor will decrease the thermodynamics quantities, whereas for $0<\kappa^{11}<1$, it will give and increasing contribution. In addition, the coefficient of proportional to $\lambda$ will decrease the thermal quantities, whereas an increasing contribution will be obtained from the term proportional to $\lambda \beta m$. Both of these contributions will depend on the sign of $\kappa^{11}$.

We can also note that in the case of $\kappa^{00} \neq 0$, there is no explicit LV contribution coming from the term proportional to $\lambda$. While in the case of $\kappa^{11} \neq 0$, this same feature occurs for the term proportional to $\beta^{2} m^{2}$.

\section{Concluding remarks}

In this paper, we have investigated Lorentz-violation (LV) effects on the thermodynamics properties of a real scalar field theory due to the presence of a constant background tensor field.

In order to find the effects of the Lorentz symmetry breaking terms on our model, we firstly looked for the particle spectrum of the theory, given explicitly in Eq. (18), from which we conclude that there should be specific bounds over the values of the $\kappa^{\mu \nu}$ coefficients in order to have a real positive energy that is also bounded from below. Then, we assumed that the magnitude of the constant tensor components are very small, i.e. $|\kappa|<<1$, in all the analysis performed, which is quite reasonable.

We have also studied the corresponding effects over relevant thermodynamics quantities, like the internal energy, pressure, and the entropy. First of all, we considered the free massless scalar field, with some specific non-vanishing coefficients for simplicity. We noticed that the effects over the thermodynamics quantities are all enclosed in a global multiplicative factor, which increases the thermodynamics quantities when the LV coefficients are positive, and decrease them when they take negative values. In particular, we found that off-diagonal non-null coefficients yield only quadratic contributions in the LV coefficients, and thus they have been neglected for our analysis.

When the massive scalar field is considered, we face in general a difficulty in order to evaluate analytically the corresponding partition function, and then only the high temperature regime $m / T<<1$ was considered. In this approximation, we have computed the corresponding thermodynamics quantities with very good accuracy. In this case, we get not only the global factor contribution, but also a contribution from the coefficient of $m^{2}$. For instance, when $0<\kappa^{00}<1$, the global factor increase the thermal quantities, whereas the square mass coefficient produces a decrease. On the other hand, when $-1<\kappa^{00}<0$, both contributions cause the pressure, internal energy and entropy to decrease. In addition, by considering only $\kappa^{11} \neq 0$, we only obtain a purely global contribution, such that when $\kappa^{11}<0$, the pressure, internal energy, and entropy decrease more rapidly than in the case of positive $\kappa^{11}$.

Subsequently, we analysed the case of a LV massless scalar field in the presence of a $\phi^{4}$ interaction potential. Interestingly, we noticed that this case is somehow similar to the free massive case, where the coupling constant $\lambda$ plays the role of the mass $m$, but with LV contributions of different magnitude. This similarity can be understood from the effect of the global factor on the thermal quantities. However, unlike the free massive case, the thermal quantities will decrease because of the interaction factor when we consider $\kappa^{11} \neq 0$.

Finally, we analysed the massive interacting scalar field theory in the LV tensor background. In this case, we find several possibilities of decreasing and increasing contributions coming from the LV coefficients. We noticed that when only $\kappa^{00} \neq 0$, there is no such Lorentz breaking coming from the term proportional to the coupling constant $\lambda$. While in the case when only $\kappa^{11} \neq 0$, this same features occur for the term proportional to $\beta^{2} m^{2}$.

We believe that the extension our results to more general $\mathrm{LV}$ models is an interesting issue that can be addressed in next works. In particular, it would be interesting to analyse low temperature behaviours, and also extensions to LV models with boundaries. Potential applications, such as LV effects on cosmological problems [73-77], or in perturbative quantum field theory [78], would also be of great interest. Authors aim to pursue these further studies in future investigations.

Acknowledgements A.R.A and G.F.H. thank to the Brazilian agency CAPES for partial financial support. E.S.S. thanks CAPES for full financial support, and R.G.R. thanks to the Brazilian research agency $\mathrm{CNPq}$ for full financial support.

Data Availability Statement This manuscript has no associated data or the data will not be deposited. [Authors' comment: All the data is available in the article and references. Therefore, data sharing is not applicable.]

Open Access This article is licensed under a Creative Commons Attribution 4.0 International License, which permits use, sharing, adaptation, distribution and reproduction in any medium or format, as long as you give appropriate credit to the original author(s) and the source, provide a link to the Creative Commons licence, and indicate if changes were made. The images or other third party material in this article are included in the article's Creative Commons licence, unless indicated otherwise in a credit line to the material. If material is not included in the article's Creative Commons licence and your intended use is not permitted by statutory regulation or exceeds the permit- 
ted use, you will need to obtain permission directly from the copyright holder. To view a copy of this licence, visit http://creativecomm ons.org/licenses/by/4.0/.

Funded by SCOAP ${ }^{3}$.

\section{Appendix A: Explicit calculation of the partition function}

Let us start from the expression (31) for the partition function for the free scalar field at finite temperature, namely

$$
\begin{aligned}
Z_{0}= & N^{\prime} \prod_{n p}\left[\beta ^ { 2 } \left(\bar{\Delta}_{00} \omega_{n}^{2}+2 \bar{\Delta}_{0 j} \omega_{n} p_{j}\right.\right. \\
& \left.\left.+\bar{\Delta}_{i j} p_{i} p_{j}+m^{2}\right)\right]^{-1 / 2}
\end{aligned}
$$

from which we straightforwardly obtain,

$$
\begin{aligned}
\ln Z_{0}= & -\frac{1}{2} \sum_{n=-\infty}^{\infty} \sum_{p} \ln \left(\beta^{2} \omega_{n}^{2}+2 \frac{\bar{\Delta}_{0 j}}{\bar{\Delta}_{00}} \beta^{2} \omega_{n} p_{j}+\frac{\beta^{2} \omega^{2}}{\bar{\Delta}_{00}}\right) \\
& +\ln N^{\prime}
\end{aligned}
$$

where we have disregarded the term that does not depend on the thermodynamics variables, and

$w^{2}=\bar{\Delta}_{i j} p_{i} p_{j}+m^{2}, \quad \omega_{n}=\frac{2 \pi n}{\beta}$.

By using (A.3), and introducing the following notation

$A=\frac{\kappa_{0 j}}{\bar{\Delta}_{00}} \beta p_{j}, \quad \theta=\frac{\beta^{2} \omega^{2}}{\bar{\Delta}_{00}}$,

the expression can be simplified to the following form

$\ln Z_{0}=\ln N^{\prime}-\frac{1}{2} \sum_{n=-\infty}^{\infty} \sum_{p} \ln \left(4 \pi^{2} n^{2}-4 \pi i A n+\theta\right)$

Now, let us derive and integrate the right-hand side of (A.5) with respect to $\theta$. Doing that, we note that the summation can be expressed as follows,

$$
\begin{aligned}
& \sum_{n=-\infty}^{\infty} \sum_{p} \int \frac{d \theta}{\left[4 \pi^{2} n^{2}-4 \pi i A n+\theta\right]} \\
& =\sum_{p} \int d \theta \sum_{n=-\infty}^{\infty} \frac{1}{\left[4 \pi^{2} n^{2}-4 \pi i A n+\theta\right]}
\end{aligned}
$$

The summation in (A.6) can be computed exactly, and the result is given by

$$
\begin{aligned}
& \sum_{n=-\infty}^{\infty} \frac{1}{\left[4 \pi^{2} n^{2}-4 \pi i A n+\theta\right]} \\
& =\frac{1}{4 \sqrt{A^{2}+\theta}}\left[\operatorname{coth}\left(\frac{A+\sqrt{A^{2}+\theta}}{2}\right)\right. \\
& \left.-\operatorname{coth}\left(\frac{A-\sqrt{A^{2}+\theta}}{2}\right)\right] .
\end{aligned}
$$

Now, by using Eq. (A.7), we can solve the integral in Eq. (A.6),

$$
\begin{gathered}
\int d \theta \sum_{n=-\infty}^{\infty} \frac{1}{\left[4 \pi^{2} n^{2}-4 \pi i A n+\theta\right]} \\
=C_{1}+\ln \left[\sinh \left(\frac{A-\sqrt{A^{2}+\theta}}{2}\right)\right] \\
+\ln \left[\sinh \left(\frac{A+\sqrt{A^{2}+\theta}}{2}\right)\right]
\end{gathered}
$$

where $C_{1}=C_{1}(\kappa, \beta)$ is an integration constant that will be used to cancel out the term $\ln N^{\prime}$ in Eq. (A.2). Then, we find

$$
\begin{aligned}
& \ln Z_{0}=-\frac{1}{2} \sum_{p}\left\{\beta \sqrt{\left(\frac{\kappa_{0 j}}{\bar{\Delta}_{00}} p_{j}\right)^{2}+\frac{\omega^{2}}{\bar{\Delta}_{00}}}\right. \\
&\left.+\ln \left[1-e^{\left.-\beta\left(\sqrt{\left(\frac{\kappa_{0 j}}{\bar{\Delta}_{00}} p_{j}\right)^{2}+\frac{\omega^{2}}{\bar{\Delta}_{00}}}-\frac{\kappa_{0 j}}{\bar{\Delta}_{00}} p_{j}\right)\right]}\right]\right] \\
&+\ln \left[1-e^{\left.-\beta\left(\sqrt{\left(\frac{\kappa_{0 j}}{\bar{\Delta}_{00}} p_{j}\right)^{2}+\frac{\omega^{2}}{\bar{\Delta}_{00}}}+\frac{\kappa_{0 j}}{\bar{\Delta}_{00}} p_{j}\right)\right]}\right]
\end{aligned}
$$

Now, we can relabel the variable $p \rightarrow-p$ in the last term of (A.9), and then simplify the above expression as follows

$$
\begin{aligned}
\ln Z_{0}= & \sum_{p}\left\{-\frac{\beta}{2} \sqrt{\left(\frac{\kappa_{0 j}}{\bar{\Delta}_{00}} p_{j}\right)^{2}+\frac{\omega^{2}}{\bar{\Delta}_{00}}}\right. \\
& -\ln \left[1-e^{\left.\left.-\beta\left(\sqrt{\left(\frac{\kappa_{0 j}}{\bar{\Delta}_{00}} p_{j}\right)^{2}+\frac{\omega^{2}}{\bar{\Delta}_{00}}}-\frac{\kappa_{0 j}}{\bar{\Delta}_{00}} p_{j}\right)\right]\right\} .} .\right.
\end{aligned}
$$


For the continuous case we will find

$$
\begin{aligned}
& \ln Z_{0}=-V \int \frac{d^{3} p}{(2 \pi)^{3}}\left\{\frac{\beta}{2} \sqrt{\left(\frac{\kappa_{0 j}}{\bar{\Delta}_{00}} p_{j}\right)^{2}+\frac{\omega^{2}}{\bar{\Delta}_{00}}}\right.
\end{aligned}
$$

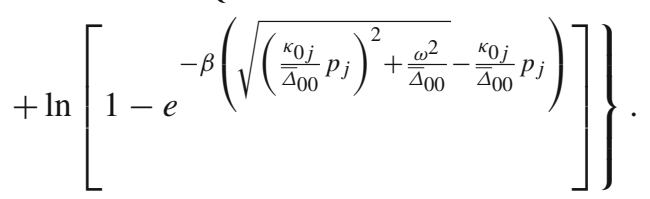

\section{Appendix B: Mean number of particle for the massive case}

Let us consider the mean number of particles for the massive case, given by

$$
N=V \int \frac{d^{3} p}{(2 \pi)^{3}} \frac{1}{e^{\beta p_{0}}-1},
$$

where $p_{0}$ is given by Eq. (18). By using Eqs. (34), (35), and considering $\kappa^{0 i}=0$ for simplicity, we get

$N=\frac{V|\operatorname{det} M| \beta^{-3}}{2 \pi^{2}|\operatorname{det} A|^{1 / 2}} \int d \bar{p} \frac{\bar{p}^{2}}{e^{\sqrt{\bar{p}^{2}+a^{2}}}-1}$,

where $\bar{p}$ is defined as in (35) and

$a^{2}=\frac{\beta^{2} m^{2}}{\left(1+\kappa^{00}\right)}$.

To properly calculate the integral in (B.2), valid for small $\beta m$, we can expand the integrand in orders of $a$. However, it is not possible to use Taylor's expansion since the integrand is not an analytic function on $a=0$, as one can see taking the first derivative of (B.2). However, we can sort out this problem by using the identity [55],

$\frac{1}{e^{y}-1}=-\frac{1}{2}+\sum_{n=-\infty}^{\infty} \frac{y}{y^{2}+4 \pi^{2} n^{2}}$,

in the integral of Eq. (B.2), we have

$$
\begin{aligned}
I(a) & =\int d \bar{p} \frac{\bar{p}^{2}}{e^{\sqrt{\bar{p}^{2}+a^{2}}}-1} \\
& =-\int d \bar{p} \frac{\bar{p}^{2}}{2}+\sum_{n=-\infty}^{\infty} \int d \bar{p} \frac{\bar{p}^{2} \sqrt{\bar{p}^{2}+a^{2}}}{\bar{p}^{2}+a^{2}+4 \pi^{2} n^{2}}+\delta I,
\end{aligned}
$$

where $\delta I$ in (B.5) is a necessary counterterm to ensure convergence of the integral $I(a)$, since the identity (B.4) gen- erates a fictitious divergence. Separating the massless terms, we can write

$$
\begin{aligned}
I(a)= & I(0)+\delta I+\sum_{n=-\infty}^{\infty}\left[\int d \bar{p} \frac{\bar{p}^{2} \sqrt{\bar{p}^{2}+a^{2}}}{\bar{p}^{2}+a^{2}+4 \pi^{2} n^{2}}\right. \\
& \left.-\int d \bar{p} \frac{\bar{p}^{3}}{\bar{p}^{2}+4 \pi^{2} n^{2}}\right] .
\end{aligned}
$$

Hence, we are concerned with calculating the last two integrals of Eq. (B.6), which we will denote as

$J=\int d \bar{p} \frac{\bar{p}^{2} \sqrt{\bar{p}^{2}+a^{2}}}{\bar{p}^{2}+a^{2}+4 \pi^{2} n^{2}}$,

and

$K=\widetilde{K}+\int d \bar{p} \frac{\bar{p}^{3}}{\bar{p}^{2}+4 \pi^{2} n^{2}}$,

where $\widetilde{K}$ is a divergent mass-independent constant, whose derivative arises from the term $n=0$. Let us begin with $J$. First, we approximate in the following way,

$$
\begin{aligned}
J \approx & \int_{0}^{\infty} d \bar{p} \frac{\bar{p}^{3}}{\bar{p}^{2}+a^{2}+4 \pi^{2} n^{2}} \\
& +\frac{a^{2}}{2} \int_{0}^{\infty} d \bar{p} \frac{\bar{p}}{\bar{p}^{2}+a^{2}+4 \pi^{2} n^{2}}
\end{aligned}
$$

and use dimensional regularization by inserting a parameter $\delta$, as follows

$$
\begin{aligned}
J \approx & \int_{0}^{\infty} d \bar{p} \frac{\bar{p}^{3-2 \delta}}{\bar{p}^{2}+a^{2}+4 \pi^{2} n^{2}} \\
& +\frac{a^{2}}{2} \int_{0}^{\infty} d \bar{p} \frac{\bar{p}^{1-\delta}}{\bar{p}^{2}+a^{2}+4 \pi^{2} n^{2}} \\
\approx & -\frac{2 \pi^{2} n^{2}}{\delta}+\left(2 \pi^{2} n^{2}+\frac{a^{2}}{4}\right) \ln \left(a^{2}+4 \pi^{2} n^{2}\right) .
\end{aligned}
$$

Similarly for the integral $K$, we obtain

$$
\begin{aligned}
K & =\widetilde{K}+\int d \bar{p} \frac{\bar{p}^{3-2 \delta}}{\bar{p}^{2}+4 \pi^{2} n^{2}} \\
& =\widetilde{K}-\frac{2 \pi^{2} n^{2}}{\delta}+2 \pi^{2} n^{2} \ln \left(4 \pi^{2} n^{2}\right)
\end{aligned}
$$

Replacing above expressions in (B.6) we note that the divergent terms of the integrals $J$ and $K$ cancel each other out, allowing us to perform the sums in (B.6), and then we find 
the following,

$$
\begin{aligned}
I(a)= & I(0)+\delta I-\widetilde{K}+\frac{a^{2}}{4} \ln \left(a^{2}\right) \\
& +\sum_{n=1}^{\infty}\left[\frac{a^{2}}{2} \ln \left(a^{2}+4 \pi^{2} n^{2}\right)\right. \\
& \left.+4 \pi^{2} n^{2} \ln \left(1+\frac{a^{2}}{4 \pi^{2} n^{2}}\right)\right] .
\end{aligned}
$$

The first sum above is computed using the identity

$$
\prod_{n=1}^{\infty}\left(1+\frac{a^{2}}{4 \pi^{2} n^{2}}\right)=\frac{2}{a} \sinh (a / 2),
$$

and the last one can be calculated for small $a$, using Taylor expansion, obtaining

$$
\begin{aligned}
& \sum_{n=1}^{\infty} n^{2} \ln \left(1+\frac{a^{2}}{4 \pi^{2} n^{2}}\right) \\
& \approx \sum_{n=1}^{\infty}\left(\frac{a^{2}}{4 \pi^{2}}-\frac{a^{4}}{32 \pi^{4} n^{2}}+\mathcal{O}\left(a^{6}\right)\right) .
\end{aligned}
$$

In this way, we get for (B.12), using the cutoff regularization

$$
\begin{aligned}
I(a)= & I(0)+\frac{a^{2}}{2} \ln \sinh (a / 2)+\mathcal{O}\left(a^{4}\right)+\delta I-\widetilde{K} \\
& +\left.a^{2}(\widetilde{C}+\ln (2 \pi \Lambda)+\Lambda)\right|_{\Lambda \rightarrow \infty},
\end{aligned}
$$

where $\widetilde{C}$ is a divergent constant. We expect that at the limit $I(a \rightarrow 0) \rightarrow I(0)$, and we find

$$
\delta I=\widetilde{K}-\lim _{a \rightarrow 0}\left[\left.a^{2}(\widetilde{C}+\ln (2 \pi \Lambda)+\Lambda)\right|_{\Lambda \rightarrow \infty}\right] .
$$

However, since we assume that the $I(a)$ function is smooth and continuous, we can generalize Eq. (B.16) for all allowed

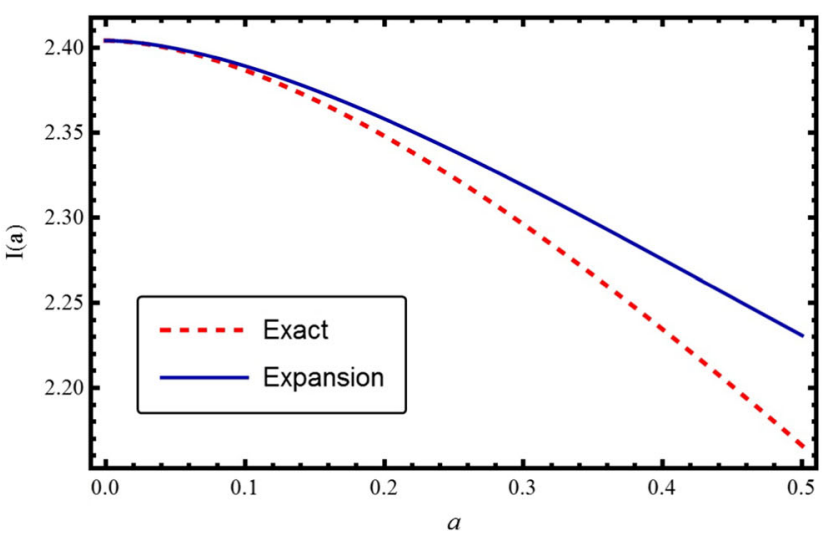

Fig. 1 On the left, we have plotted the values of the integral both exact numerical calculation (red dashed line) and the approximation (blue continuous line). On the right, we have plotted the relative error of values of $a$, i.e., those that make sense in an expansion of $a$. Thus, we have

$I(a)=I(0)+\frac{a^{2}}{2} \ln \sinh (a / 2)+\mathcal{O}\left(a^{4}\right)$.

In Fig. 1, we have plotted the values of the integral both exact numerical calculation, and approximated calculation, and the corresponding relative error. We can notice that the relative error will be less than $1 \%$ when compared with the numerical value, for $a \lesssim 0.3$. From these numerical support, we can ensure that the approximated value for the mean number of particles is reliable.

Finally, by substituting our approximation in (B.2), we can write down the mean number of particles formula for the massive case in the following form,

$$
\begin{aligned}
N= & \frac{V|\operatorname{det} M| T^{3}}{\pi^{2}|\operatorname{det} A|^{1 / 2}} \\
& \times\left(\zeta(3)+\frac{m^{2} T^{-2}}{4\left(1+\kappa^{00}\right)} \ln \sinh \left(\frac{m}{2 \sqrt{\left(1+\kappa^{00}\right)} T}\right)\right. \\
& \left.+\mathcal{O}\left((m / T)^{4}\right)\right) .
\end{aligned}
$$

\section{Appendix C: First correction for the massive interacting partition function}

In this appendix we compute the integral in (73) for the massive case. Using (34) and the change of variable (35), we have

$$
\begin{aligned}
& \int \frac{d^{3} \mathbf{p}}{(2 \pi)^{3}} \frac{1}{\left[\left(1+\kappa^{00}\right) p_{0}+\kappa^{0 j} p_{j}\right]\left(e^{\beta p_{0}}-1\right)} \\
& =\frac{|\operatorname{det} M| \beta^{-2}}{8 \pi^{3}|\operatorname{det} A|^{1 / 2}\left(1+\kappa^{00}\right)} \tilde{I}(a),
\end{aligned}
$$

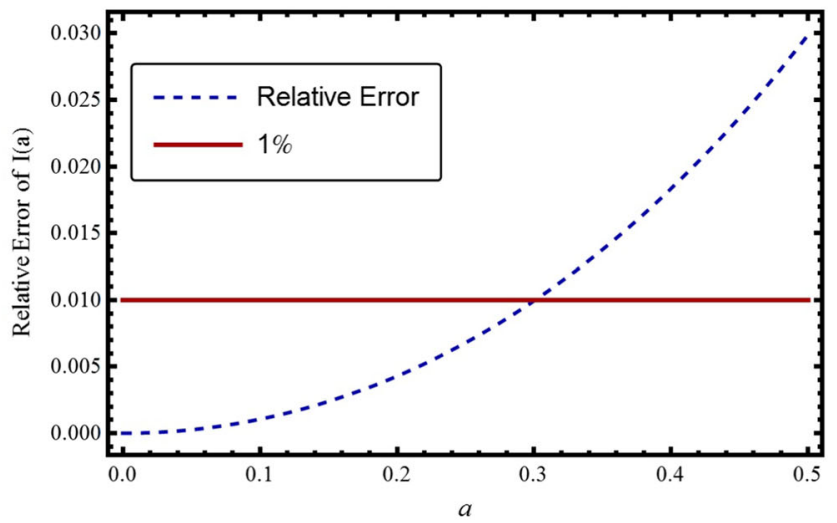

the approximated value of the integral with respect to numerical exact value. The horizontal continuous (red) line corresponds to an error of $1 \%$ 
where

$$
\tilde{I}(a)=\int d^{3} \overline{\mathbf{p}} \frac{1}{\sqrt{\bar{p}^{2}+a^{2}}\left[\exp \left(\sqrt{\bar{p}^{2}+a^{2}}-\mathbf{b} \cdot \overline{\mathbf{p}}\right)-1\right]},
$$

where $\mathbf{b}$ and $a^{2}$ are given respectively by Eqs. (37) and (B.3). Integral (C.2) can not be computed exactly, and then here we will an approximated expression valid for sufficiently small $a$. For this purpose, we first integrate in spherical coordinates, taking the polar axis along the vector $\mathbf{b}$. In this way we get,

$$
\begin{aligned}
\tilde{I}(a) & =2 \pi \int_{0}^{\pi} d \theta \int_{0}^{\infty} d \bar{p} \frac{\sin (\theta) \bar{p}^{2}\left(\bar{p}^{2}+a^{2}\right)^{-1 / 2}}{\exp \left(\sqrt{\bar{p}^{2}+a^{2}}-\cos (\theta) b \bar{p}\right)-1} \\
& =2 \pi \int_{-1}^{1} d \tau \int_{0}^{\infty} d \bar{p} \frac{\bar{p}^{2}\left(\bar{p}^{2}+a^{2}\right)^{-1 / 2}}{\exp \left(\sqrt{\bar{p}^{2}+a^{2}}-b \bar{p} \tau\right)-1},
\end{aligned}
$$

where we have used the change of variable $\tau=\cos (\theta)$. Using the identity (B.4) in (C.3), we get

$$
\begin{aligned}
\tilde{I}(a)= & -2 \pi \int_{0}^{\infty} d \bar{p} \frac{\bar{p}^{2-\epsilon}}{\sqrt{\bar{p}^{2}+a^{2}}}+2 \pi \sum_{n=-\infty}^{\infty} \int_{-1}^{1} d \tau \int_{0}^{\infty} d \bar{p}\left(\bar{p}^{2-\epsilon}\right) \\
& \times \frac{\left(\bar{p}+a^{2}\right)^{-1 / 2}\left(\sqrt{\bar{p}^{2}+a^{2}}-b \bar{p} \tau\right)}{\left(\sqrt{\bar{p}^{2}+a^{2}}-b \bar{p} \tau\right)^{2}+4 \pi^{2} n^{2}}+\delta \tilde{I},
\end{aligned}
$$

where a regulator $(\bar{p})^{-\epsilon}$ is introduced to give sense to the change of the order between the sum and the integral. This change also introduces an ambiguity, that we denote as $\delta \tilde{I}$, and will be fixed with the known value of $\tilde{I}$ at $a=0$. For this value, we get easily from (C.3)

$\tilde{I}(0)=\frac{2 \pi^{3}}{3\left(1-b^{2}\right)}$.

Now, for sufficiently small $a$, after expansion of the square root in (C.4), we get

$\tilde{I}(a)=I_{1}+I_{2}+I_{3}+\delta \tilde{I}$,

where

$$
\begin{aligned}
I_{1}= & -2 \pi \int_{0}^{\infty} d \bar{p} \frac{\bar{p}^{2-\epsilon}}{\sqrt{\bar{p}^{2}+a^{2}}}, \\
I_{2}= & 2 \pi \sum_{n=-\infty}^{\infty} \int_{-1}^{1} d \tau(1-b \tau) \\
& \times \int_{0}^{\infty} d \bar{p} \frac{\bar{p}^{2-\epsilon}}{(1-b \tau)^{2} \bar{p}^{2}+(1-b \tau) a^{2}+4 \pi^{2} n^{2}},
\end{aligned}
$$

and

$$
\begin{aligned}
I_{3}= & \pi b a^{2} \sum_{n=-\infty}^{\infty} \int_{-1}^{1} d \tau \tau \\
& \times \int_{0}^{\infty} d \bar{p} \frac{\bar{p}^{-\epsilon}}{(1-b \tau)^{2} \bar{p}^{2}+(1-b \tau) a^{2}+4 \pi^{2} n^{2}} .
\end{aligned}
$$

The integral (C.7) can be evaluated in terms of the Beta function, namely

$$
\begin{aligned}
I_{1} & =-2 \pi a^{2-\epsilon} B\left(\frac{3}{2}-\frac{\epsilon}{2},-1+\frac{\epsilon}{2}\right) \\
& =\frac{\pi a^{2}}{\epsilon}-\frac{\pi a^{2}}{2}(1+2 \ln (a / 2)) .
\end{aligned}
$$

The integrals (C.8) and (C.9) are evaluated using an appropriated change of variable and

$\int_{0}^{\infty} d x \frac{x^{-\alpha}}{x^{2}+1}=\frac{\pi}{2 \cos (\pi \alpha / 2)}$

In this way get respectively

$I_{2}=-\pi^{2} \sum_{n=-\infty}^{\infty} \int_{-1}^{1} d \tau \frac{\left[(1-b \tau) a^{2}+4 \pi^{2} n^{2}\right]^{(1-\epsilon) / 2}}{(1-b \tau)^{2-\epsilon} \cos (\pi \epsilon / 2)}$,

and

$I_{3}=\frac{\pi^{2} a^{2} b}{2} \sum_{n=-\infty}^{\infty} \int_{-1}^{1} d \tau \frac{\tau\left[(1-b \tau) a^{2}+4 \pi^{2} n^{2}\right]^{-(1+\epsilon) / 2}}{(1-b \tau)^{1-\epsilon} \cos (\pi \epsilon / 2)}$.

Now, taking the limit $\epsilon \rightarrow 0$, separating the term $n=0$, and expanding in powers of $a^{2}$, we obtain for (C.12)

$$
\begin{aligned}
I_{2}= & -\pi^{2} a \int_{-1}^{1} d \tau \frac{1}{(1-b \tau)^{3 / 2}}-8 \pi^{3} \frac{\zeta(-1)}{1-b^{2}} \\
& -\frac{\pi a^{2}}{2}\left(\frac{1}{\epsilon}+\gamma-\ln 2 \pi\right) \int_{-1}^{1} d \tau \frac{1}{1-b \tau}+\cdots,
\end{aligned}
$$

where $\gamma$ is the Euler-Mascheroni constant. In a similar way, we get for (C.14)

$$
\begin{aligned}
I_{3}= & \frac{\pi^{2} a b}{2} \int_{-1}^{1} d \tau \frac{\tau}{(1-b \tau)^{3 / 2}} \\
& +\frac{\pi a^{2}}{2}\left(\frac{1}{\epsilon}+\gamma-\ln 2 \pi\right) \int_{-1}^{1} d \tau \frac{b \tau}{1-b \tau}+\cdots .
\end{aligned}
$$


Replacing (C.10), (C.14) and (C.15) in (C.6), we see that the divergent terms at $\epsilon \rightarrow 0$ cancel out, and we obtain

$$
\begin{aligned}
\tilde{I}(a)= & \frac{\pi^{2} a}{2} \int_{-1}^{1} d \tau \frac{b \tau-2}{(1-b \tau)^{3 / 2}}-\frac{8 \pi^{3} \zeta(-1)}{1-b^{2}} \\
& -\frac{\pi a^{2}}{2}\left(1+2 \gamma+2 \ln \frac{a}{4 \pi}\right)+\delta \tilde{I} .
\end{aligned}
$$

Now we can fix $\delta \tilde{I}$. Taking $a \rightarrow 0$ in expression above we find

$\delta \tilde{I}=\frac{8 \pi^{3} \zeta(-1)}{1-b^{2}}+\tilde{I}(0)$.

Using the above result, and performing the integration in $\tau$, we obtain

$$
\begin{aligned}
\tilde{I}(a)= & \frac{2 \pi^{3}}{3\left(1-b^{2}\right)}-\pi^{2} a\left(\frac{1}{\sqrt{1-b}}+\frac{1}{\sqrt{1+b}}\right) \\
& -\frac{\pi a^{2}}{2}\left(1+2 \gamma+2 \ln \frac{a}{4 \pi}\right)+\cdots .
\end{aligned}
$$

Finally, by using Eqs. (C.18) and (C.1) in Eq. (73), we get the expression (80) for the partition function.

\section{References}

1. V.A. Kostelecký, S. Samuel, Phenomenological gravitational constraints on strings and higher-dimensional theories. Phys. Rev. Lett. 63, 224 (1989)

2. V.A. Kostelecký, S. Samuel, Gravitational phenomenology in higher-dimensional theories and strings. Phys. Rev. D 40, 1886 (1989)

3. V.A. Kostelecký, S. Samuel, Spontaneous breaking of Lorentz symmetry in string theory. Phys. Rev. D 39, 683 (1989)

4. S. Carroll, G.B. Field, R. Jackiw, Limits on a Lorentz- and parityviolating modification of electrodynamics. Phys. Rev. D 41, 1231 (1990)

5. V.A. Kostelecký, R. Potting, CPT and strings. Nucl. Phys. B 359, 545 (1991)

6. V.A. Kostelecký, R. Potting, Expectation values, Lorentz invariance, and CPT in the open bosonic string. Phys. Lett. B 381, 89 (1996)

7. D. Colladay, V.A. Kostelecký, CPT violation and the standard model. Phys. Rev. D 55, 6760 (1997). arXiv:hep-ph/9703464

8. D. Colladay, V.A. Kostelecký, Lorentz-violating extension of the standard model. Phys. Rev. D 58, 116002 (1998). arXiv:hep-ph/9809521

9. V.A. Kostelecký, N. Russell, Data tables for Lorentz and CPT violation. Rev. Mod. Phys. 83, 1 (2011). arXiv:0801.0287v14

10. D. Colladay, P. McDonald, Statistical mechanics and Lorentz violation. Phys. Rev. D 70, 125007 (2004)

11. A.A.A. Filho, R.V. Maluf, Thermodynamic properties in higherderivative electrodynamics. Braz. J. Phys. 51, 820 (2021)

12. A.A.A. Filho, Lorentz-violating scenarios in a thermal reservoir. Eur. Phys. J. Plus 136, 417 (2021)

13. A.A.A. Filho, J.A.A.S. Reis, Thermal aspects of interacting quantum gases in Lorentz-violating scenarios. Eur. Phys. J. Plus 136, $310(2021)$

14. A.A.A. Filho, A. Yu Petrov, Higher-derivative Lorentz-breaking dispersion relations: a thermal description. arXiv:2103.08738
15. M.B. Cruz, E.R. Bezerra de Mello, A. Yu Petrov, Casimir effects in Lorentz-violating scalar field theory. Phys. Rev. D 96, 045019 (2017)

16. C.A. Escobar, L. Medel, A. Martín-Ruiz, Casimir effect in Lorentzviolating scalar field theory: a local approach. Phys. Rev. D 101, 095011 (2020)

17. C.A. Escobar, A. Martín-Ruiz, O.J. Franca, M.A.G. García, A nonperturbative approach to the scalar Casimir effect with Lorentz symmetry violation. Phys. Lett. B 807, 135567 (2020)

18. M.B. Cruz, E.R. Bezerra de Mello, H.F. Santana Mota, Casimir energy and topological mass for a massive scalar field with Lorentz violation. Phys. Rev. D 102, 045006 (2020)

19. A. Mojavezi, R. Moazzemi, M.E. Zomorrodian, NLO radiative correction to the Casimir energy in Lorentz-violating scalar field theory. Nucl. Phys. B 941, 145 (2019)

20. A. Erdas, Casimir effect of a Lorentz-violating scalar in magnetic field. Int. J. Mod. Phys. A 35, 2050209 (2020)

21. D. Colladay, P. McDonald, Bose-Einstein condensates as a probe for Lorentz violation. Phys. Rev. D 73, 105006 (2006)

22. E. Castellanos, A. Camacho, Stability of Bose-Einstein condensates in a Lorentz violating scenario. Mod. Phys. Lett. A 25, 459 (2010)

23. R. Casana, K.A. da Silva, Lorentz-violating effects in the BoseEinstein condensation of an ideal bosonic gas. Mod. Phys. Lett. A 30, 1550037 (2015)

24. M.B. Cruz, E.R. Bezerra de Mello, A. Yu Petrov, Thermal corrections to the Casimir energy in a Lorentz-breaking scalar field theory. Mod. Phys. Lett. A 33, 1850115 (2018)

25. J. Furtado, A.C.A. Ramos, J.F. Assunção, Effects of Lorentz violation in the Bose-Einstein condensation. EPL (Europhys. Lett.) 132, 31001 (2020)

26. R. Casana, M.M. Ferreira Jr., J.S. Rodrigues, M.R.O. Silva, Finite temperature behavior of the CPT-even and parity-even electrodynamics of the standard model extension. Phys. Rev. D 80, 085026 (2009). arXiv:0907.1924v3

27. R. Casana, M.M. Ferreira Jr., M.R.O. Silva, Parity-odd and CPTeven electrodynamics of the SME at finite temperature. Phys. Rev. D 81, 105015 (2010). arXiv:0910.3709v1

28. M. Gomes, T. Mariz, J.R. Nascimento, A.Y. Petrov, A.F. Santos, A.J. da Silva, Free energy of Lorentz-violating QED at high temperature. Phys. Rev. D 81, 045013 (2010). arXiv:0910.4560 [hep-th]

29. R. Bufalo, Lorentz-violating effects in three-dimensional QED. Int. J. Mod. Phys. A 29, 1450112 (2014). arXiv:1408.2915 [hep-th]

30. C.A. Escobar, M.A.G. Garcia, The full CPT-even photon sector of the standard model extension at finite temperature. Phys. Rev. D 92(2), 025034025034 (2015). arXiv:1505.00069 [hep-th]

31. L.H.C. Borges, F.A. Barone, A perfectly conducting surface in electrodynamics with Lorentz symmetry breaking. Eur. Phys. J. C 77, 693 (2017)

32. L.H.C. Borges, A.F. Ferrari, F.A. Barone, Mirrors and field sources in a Lorentz-violating scalar field theory. Nucl. Phys. B 954, 114974 (2020)

33. L.H.C. Borges, F.A. Barone, New effects in the vicinity of a perfectly conducting plate in a non-minimal Lorentz violation scenario. Braz. J. Phys. 50, 647-657 (2020)

34. L.H.C. Borges, F.A. Barone, Field sources in a CPT-even Lorentzviolation Maxwell electrodynamics. Braz. J. Phys. 49, 571 (2019)

35. T. Mariz, J.R. Nascimento, E. Passos, R.F. Ribeiro, F.A. Brito, A remark on Lorentz violation at finite temperature. JHEP 10, 019 (2005). arXiv:hep-th/0509008

36. M. Gomes, J.R. Nascimento, E. Passos, AYu. Petrov, A.J. da Silva, Induction of the four-dimensional Lorentz-breaking non-Abelian Chern-Simons action. Phys. Rev. D 76, 047701 (2007)

37. J.R. Nascimento, E. Passos, AYu. Petrov, Lorentz-CPT violation, radiative corrections and finite temperature. JHEP 06, 016 (2007) 
38. E. Passos, A.Y. Petrov, Two-dimensional Lorentz-violating Chern-Simons-like action. Phys. Lett. B 662, 441 (2008). arXiv:0801.4946 [hep-th]. [Erratum: Phys. Lett. B 664, 318 (2008)]

39. F.A. Brito, L.S. Grigorio, M.S. Guimaraes, E. Passos, C. Wotzasek, Lorentz-violating Chern-Simons action under high temperature in massless QED. Phys. Lett. B 681, 495 (2009). arXiv:0906.2786 [hep-th]

40. R. Casana, M.M. Ferreira, R.V. Maluf, F.E.P. dos Santos, Effects of a CPT-even and Lorentz-violating nonminimal coupling on the electron-positron scattering. Phys. Rev. D 86, 125033 (2012). arXiv:1212.6230 [hep-th]

41. G.P. de Brito, J.T. Guaitolini Junior, D. Kroff, P.C. Malta, C. Marques, Lorentz violation in simple QED processes. Phys. Rev. D 94, 056005 (2016). arXiv:1605.08059 [hep-ph]

42. A.F. Santos, F.C. Khanna, Lorentz violation in Bhabha scattering at finite temperature. Phys. Rev. D 95, 125012 (2017)

43. A.F. Santos, F.C. Khanna, Lorentz violation, Möller scattering and finite temperature. Adv. High Energy Phys. 2018, 4596129 (2018). arXiv:1804.08566 [hep-th]

44. P.R.A. Souza, A.F. Santos, S.C. Ulhoa, F.C. Khanna, On Lorentz violation in $e^{+}+e^{-} \rightarrow \mu^{+}+\mu^{-}$scattering at finite temperature. Phys. Lett. B 791, 195 (2019)

45. B.R. Edwards, V.A. Kostelecký, Riemann-Finsler geometry and Lorentz-violating scalar fields. Phys. Lett. B 786, 319 (2018)

46. D. Colladay, L. Law, Spontaneous CPT breaking and fermion propagation in the Schwarzschild geometry. Phys. Lett. B 795, 457 (2019)

47. M.S. Berger, V. Alan Kostelecký, Supersymmetry and Lorentz violation. Phys. Rev. D 65, 091701 (2002)

48. S.G. Nibbelink, M. Pospelov, Lorentz violation in supersymmetric field theories. Phys. Rev. Lett. 94, 081601 (2005)

49. H. Belich, L.D. Bernald, P. Gaete, J.A. Helayël-Neto, F.J.L. Leal, Aspects of CPT-even Lorentz-symmetry violating physics in a supersymmetric scenario. Eur. Phys. J. C 75, 291 (2015). arXiv:1502.06126 [hep-th]

50. P.A. Ganai, O. Ahmad, J.P. Tobia, A. Fennell, V. Vyas, Lorentz symmetry breaking in supersymmetric quantum electrodynamics. Int. J. Geom. Methods Mod. Phys. 17, 2050038 (2020)

51. L.D. Bernal, P. Gaete, Y.P.M. Gomes, J.A. Helayël-Neto, Lorentzsymmetry violating physics in a supersymmetric scenario in $(2+1)$ D. EPL 129, 11005 (2020). arXiv:1912.00523 [hep-th]

52. V.A. Kostelecký, Developments in Lorentz and CPT Violation, in Proceedings of the Eighth Meeting on CPT and Lorentz Symmetry (CPT'19), Indiana University, Bloomington (2019)

53. D.A. Kirzhnits, A.D. Linde, Macroscopic consequences of the Weinberg model. Phys. Lett. B 42, 471 (1979)

54. A.D. Linde, Fate of the false vacuum at finite temperature: theory and applications. Phys. Lett. B 100, 37 (1981)

55. L. Doland, R. Jackiw, Symmetry behaviour at finite temperature. Phys. Rev. D 9, 3320 (1974)

56. S. Weinberg, Gauge and global symmetries at high temperature. Phys. Rev. D 9, 3357 (1974)

57. D.A. Kirzhnits, A.D. Linde, Symmetry behavior in gauge theories. Ann. Phys. 101, 195 (1976)

58. D.J. Gross, R.D. Pisarski, L.G. Yaffe, Rev. Mod. Phys. 53, 43 (1981)

59. A.A. Anselm, Classical states of the chiral field and nuclear collisions at very high-energy. Phys. Lett. B 217, 169 (1989)

60. A.A. Anselm, M.G. Ryskin, Production of classical pion field in heavy ion high-energy collisions. Phys. Lett. B 266, 482 (1991)

61. J.D. Bjorken, A full acceptance detector for SSC physics at low and intermediate mass scales: an expression of interest to the SSC. Int. J. Mod. Phys. A 7, 4189 (1992)

62. K. Rajagopal, F. Wilczek, Static and dynamic critical phenomena at a second order QCD phase transition. Nucl. Phys. B 399, 395 (1993). arXiv:hep-ph/9210253
63. J.I. Kapusta, Finite Temperature Field Theory (Cambridge University Press, Cambridge, 1989)

64. A. Das, Finite Temperature Field Theory (World Scientific, Singapore, 1997)

65. J. Zinn-Justin, Quantum Field Theory and Critical Phenomena, vol. 113 (Clarendon Press, Oxford, 2002)

66. M. Le Bellac, Thermal Field Theory (Cambridge University Press, Cambridge, 2000)

67. M. Quiros, Finite temperature field theory and phase transitions, in Proceedings, Summer School in High-Energy Physics and Cosmology: Trieste, Italy, 1999 (1998), pp. 187-259. arXiv:9901312 [hep-ph]

68. V. Alan Kostelecký, R. Lehnert, Stability, causality, and Lorentz and CPT violation. Phys. Rev. D 63, 065008 (2001)

69. R. Potting, Källén-Lehmann representation for Lorentz-violating field theory. Phys. Rev. D 85, 045033 (2012)

70. V.A. Kostelecký, A.C. Melissinos, M. Mewes, Searching for photon-sector Lorentz violation using gravitational-wave detectors. Phys. Lett. B 761, 1 (2016)

71. A.I. Hernández-Juárez, J. Montaño, H. Novales-Sánchez, M. Salinas, J.J. Toscano, O. Vázquez-Hernández, One-loop structure of the photon propagator in the standard model extension. Phys. Rev. D 99, 013002 (2019)

72. A. Ferrero, B. Altschul, Renormalization of scalar and Yukawa field theories with Lorentz violation. Phys. Rev. D 84, 065030 (2011)

73. S. Kanno, Jiro Soda, Lorentz violating inflation. Phys. Rev. D 74(6), 063505 (2006). arXiv:hep-th/0604192

74. P.P. Avelino, D. Bazeia, L. Losano, R. Menezes, J.J. Rodrigues, Impact of Lorentz violation on the dynamics of inflation. Phys. Rev. D 79, 123503 (2009). arXiv:0903.5297

75. F.P. Arianto, B.E. Zen, Triyanta Gunara, Supardi, Some impacts of Lorentz violation on cosmology. JHEP 09048 (2007). arXi:0709.3688

76. D. Blas, S. Sibiryakov, Technically natural dark energy from Lorentz breaking. JCAP 07, 026 (2011). arXiv:1104.3579

77. C. Armendariz-Picon, Could dark energy be vector-like? JCAP 07, 007 (2004). arXiv:astro-ph/0405267

78. M. Visser, Lorentz symmetry breaking as a quantum field theory regulator. Phys. Rev. D 80, 025011 (2009). arXiv:0902.0590 